\title{
Epidemiology, Geographic Location
}

National Cancer Institute

\section{Source}

National Cancer Institute. Epidemiology, Geographic Location. NCI Thesaurus. Code C18770.

The consideration of geographic location as a variable in disease incident, transmission, and control. 